\title{
Using the Spindle Cooling Temperature as a Tool for Compensating the Thermal Deformation of Machines
}

J. Vyroubal

Thermal error compensation of machine tools is a relatively complex problem nowadays. Machine users have very high expectations, and it is necessary to use all means to improve the cutting accuracy of existing machines. This paper deals with a novel approach, which combines standard temperature measurement of a machine tool and new temperature measurement of the spindle cooling liquid. A multinomial regression equation is then used to calculate the compensation correction of the position of the tool. This calculation does not critically overload the control system of the machines, so no external computing hardware is required. The cooling liquid approach improves the accuracy of the machine tool over an operational time of several hours.

Keywords: Machine tool, thermal compensation.

\section{Introduction}

From the point of view of temperature measurements, machine tools can be divided into two groups. The first group consists of so-called intelligent (advanced) machines, in which all required sensors are implanted directly within machine production. A machine spindle with temperature sensors built in to the bearings, motor, etc., is a typical representative of this group. This machine can solve deformation problems using mechatronic methods. Unfortunately, these machines are not very common. They are also relatively expensive to produce. The second group comprises conventional machines that have only a limited number of built-in sensors (about five). These sensors are specially added to the surface of the machine frame. The spindle is generally not sufficiently monitored. Machines of this type are preferred nowadays [1, 2 and 3$]$.

Conventional machine tools can be supplemented by additional sensors. However, placement of the sensors can be extremely problematic, see [4]. The problems can be summed as follows:

- The sensor cannot be placed directly inside the heat source.

- The sensor cannot be placed in the correct position because of the equipment and structure of the machine.

- The sensor is too big for the chosen measuring place.

- The sensor cannot be sunk into the material.

- The contact surface between the sensor and the frame is not perfect.

An example of sensor mounting is shown in Fig. 1.

These problems are most visible in spindle thermal behavior analysis. As mentioned, it is not possible to disassemble the spindle. In addition, the spindle is not designed for installing additional elements, due to its very sophisticated inner structure. Nowadays the most commonly applied option is to install the required sensors on the spindle head, as close as possible to the spindle. Another option is to place the sensors on the spindle tube. In terms of heat generation, the spindle is

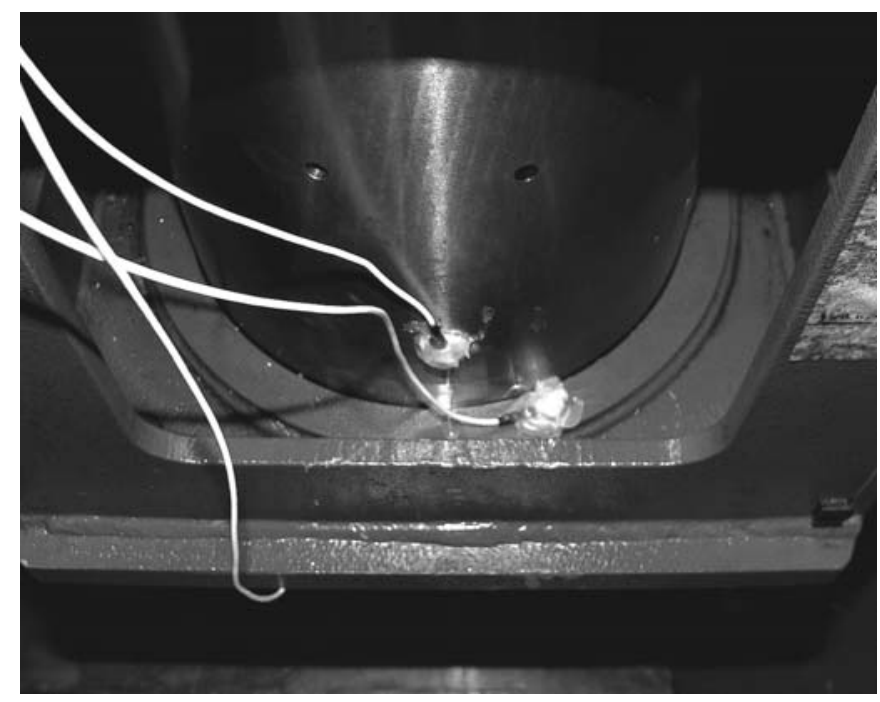

Fig. 1: Example of sensor mounted on a spindle

the major source of heat, and its thermal deformation is a major cause of overall machine deformation. This effect is multiplied when an electro-spindle with an integrated electro-motor is used. The high deformation of this type of spindle is caused by its typical mechanical structure with a group of front and rear bearings and the electro-motor winding. It is placed between the two bearing groups.

These three parts of the spindle form a considerable source of heat, but they are covered by other spindle mass (lubricating and cooling circuits, spindle tube, etc.). If the sensors are placed on the outer surface of the spindle tube, there is a relatively large traffic time delay of the transferred heat on the way out from the heat source to the temperature sensors. This delay can cancel the thermal compensation of machine deformation. The sensors fail to react when the spindle (and also the machine frame) is already deformed by heat [5]. The cutting accuracy is lower than expected. The effort to eliminate this negative effect is a basic challenge for engineers working on machine tools. An unexplored approach to the problem is to use the spindle cooling liquid as a carrier of information about the thermal state inside the spindle. 

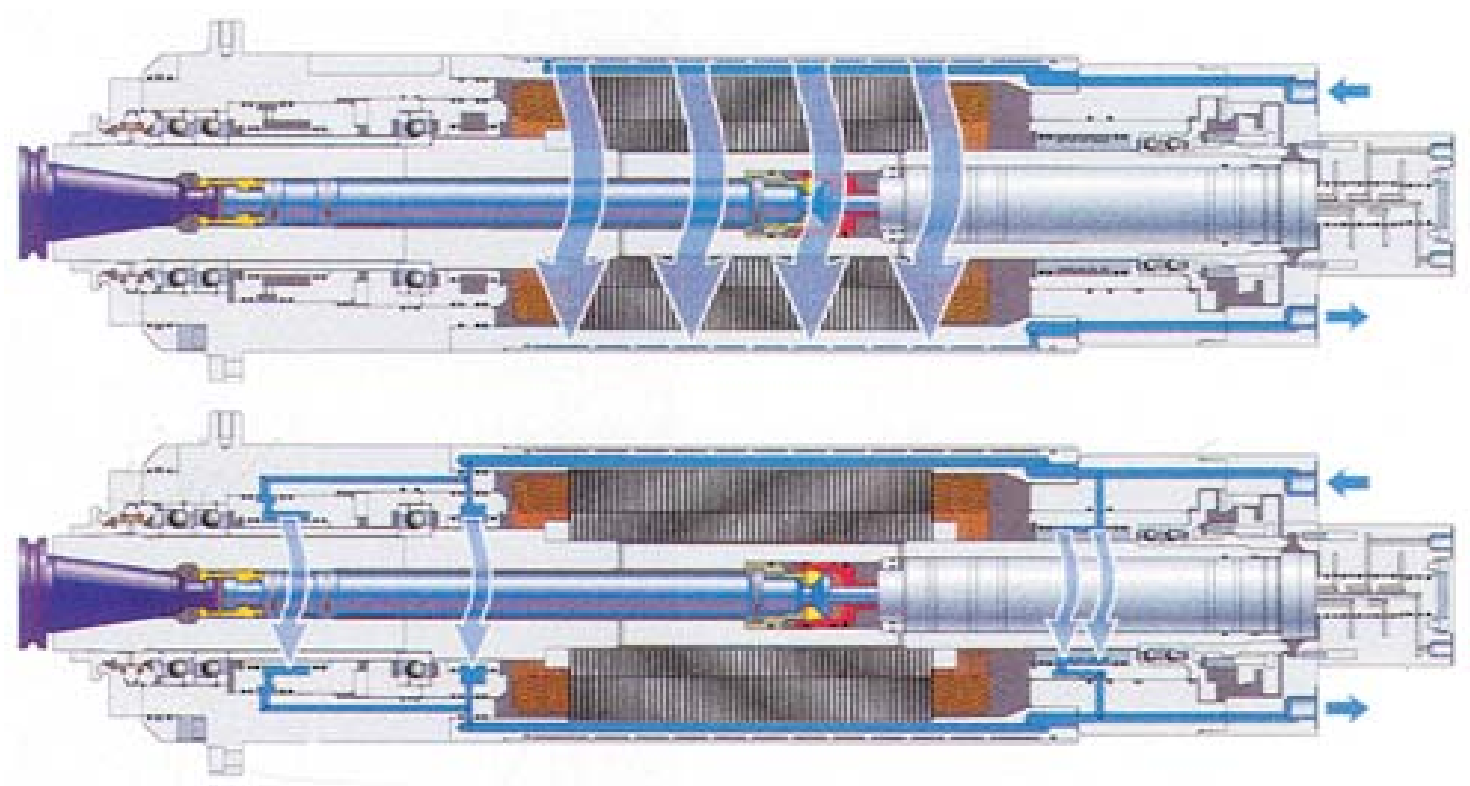

Fig. 2: Cooling circuits of the tested spindle

\section{Making use of the spindle cooling liquid}

Our task is to find a method for obtaining information about the inner thermal behavior of the spindle by measuring the temperature outside the spindle. The only option is to use the spindle cooling liquid. This liquid flows close around the groups of bearings and around the electromotor - Fig. 2. It removes the generated heat by circulating through the spindle. If the temperature sensor is placed in the liquid tube at the exit of the spindle, it can determine the conditions inside the spindle. An advantage of this measurement is speed with which the liquid takes temperature information from the bearings to the sensor. This delivery time is shorter than the time taken by heat passing through the mass of material, from the bearings to the outer surface of the spindle, where sensors are standardly placed.

Our experiments prove, that the reaction of the sensor to the temperature change is much faster (in the case of outgoing spindle cooling liquid) than for other sensors installed on the machine frame. The time results are given below.

\section{Machine tool thermal behavior monitoring}

Experiments aimed at verifying our hypothesis were performed on the MCFV 5050LN 3-axis machining center, equipped with an electro-spindle and a linear motor in all three axes. This machine has a type $\mathrm{C}$ frame, the most common type of frame. The goal of our project was to eliminate the thermal deformation of the vertical Z-axis, caused by the spindle. The overall machine deformation was monitored in the place of the tool in the direction of the Z-axis.

The machine was repeatedly thermally loaded by the rotation of the spindle. The analysis began from the cool state, after the machine had been switched off 48 hours before the experiment. In this way, the machine was brought to room tem- perature. Then the machine was started, referenced and spindle was set in motion at a constant rotation speed of $7500 \mathrm{rpm}$ $\left(50 \% n_{\max }\right)$.

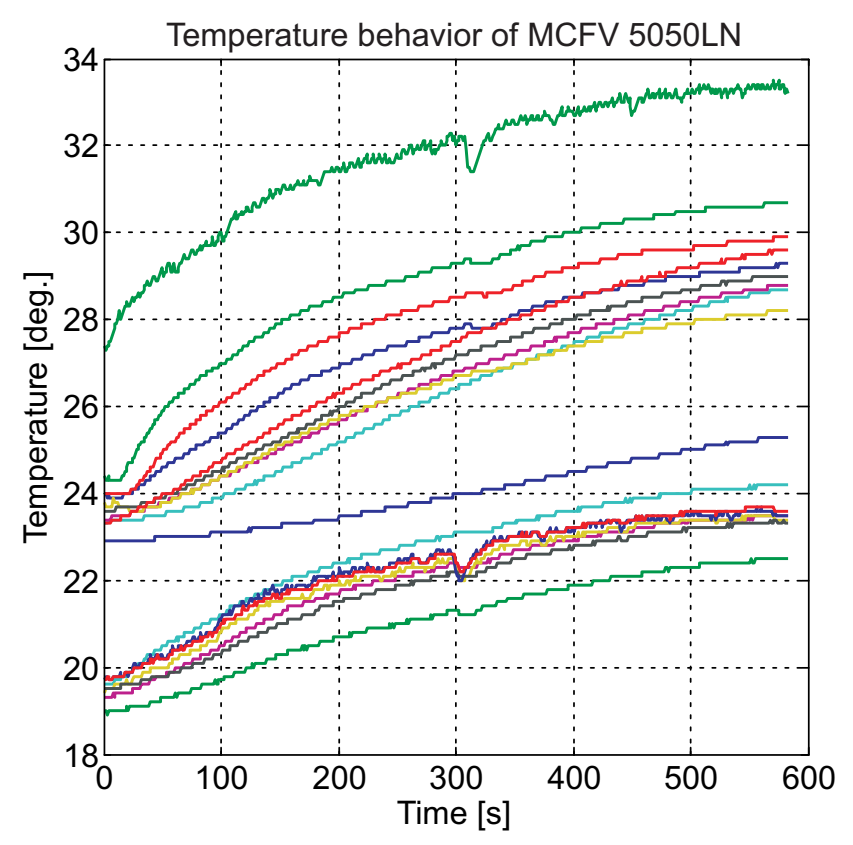

Fig. 3: Thermal behavior of MCFV 5050LN

Fig. 3. shows that the test ran for about 10 hours. This warmed the structure sufficiently to show the direction and amount of heat flux flowing from the spindle to the machine frame. The deformation of this type of thermal load in the $\mathrm{Z}$ direction is shown in Fig. 4. The initial very fast phase is caused by the spindle itself. The middle phase, between "50 min" and "150 min" is a mixture of influences from the spindle and from the column deformation. The last phase, from " 150 min" is created by the column only.

Another problem in implementing a compensation mechanism for the machine is its cost. It is necessary to offer solu- 


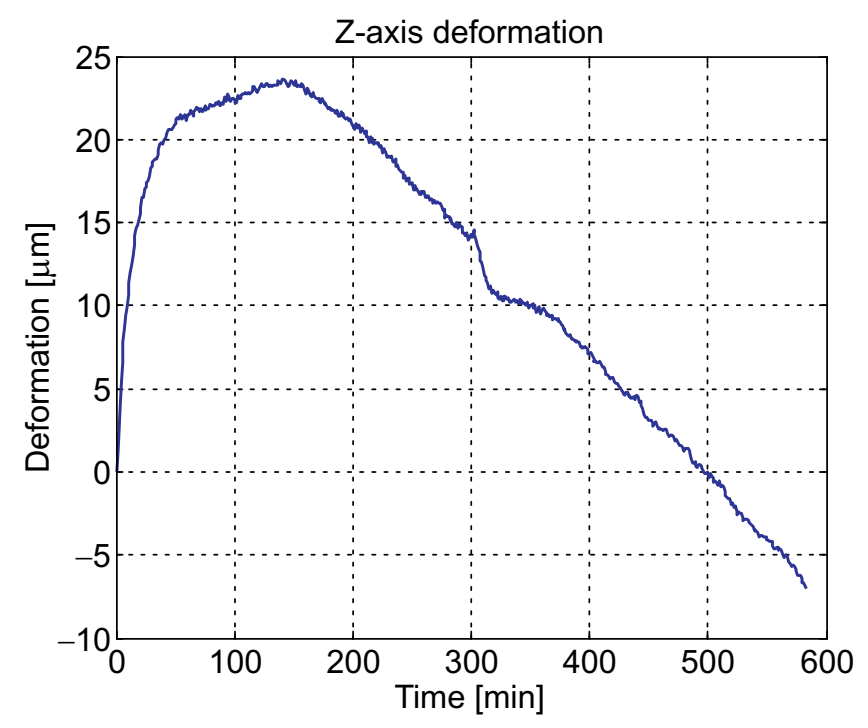

Fig. 4: Z-axis deformation in the place of the tool

tions that are inexpensive but still function well . A typical solution is a multiregression analysis. Even without additional hardware, the machine control system is not overloaded.

\section{Multiregression compensation}

Multiregression compensation is based on the principle of results calculated from several inputs. These can be written as equation:

$$
\delta=\sum_{n=1}^{m} a_{n} \cdot T_{n} .
$$

$\delta \quad$ calculated deformation,

$T$ measured temperatures,

a calculated coefficients,

$m$ number of used sensors.

For a better overview of the complete thermal behavior, of the machine, many sensors are installed on the frame and spindle. The sensors are selected by comparative analysis, using two parameters:

The first parameter is a match between increased deformation and increased temperature at a specific place.

The second parameter is the reaction speed to the defined temperature change of the measured place. The reaction limit was set up to $0.5 \mathrm{deg}$ for this experiment. Four sensors were chosen. The sensors covered the spindle ( 2 sensors), the vertical machine column and the influence of the $\mathrm{Z}$-axis linear motor. The sensors are shown in Table 1, and their placement is shown in Fig. 5.

The temperature data serve as an input for proper compensation (Eq. 1) through the machine control system. The result of Eq. 1, calculated in a given time cycle, presents a correction signal for the control system of the machine. Instead of the time-deformation characteristic, the special temperature deformation characteristic is used to form multinomial compensation. The heating process can vary in time but from the physical point of view the temperature change is dominant for the deformation magnitude.
Table 1: Sensors and their reaction time

\begin{tabular}{|c|c|}
\hline Senzors & Time reaction \\
\hline C10 & $32 \mathrm{~min}$ \\
\hline C9 & $25 \mathrm{~min}$ \\
\hline A67 & $3 \mathrm{~min}$ \\
\hline C11 & $8 \mathrm{~min}$ \\
\hline 0086 & $6 \mathrm{~min}$ \\
\hline
\end{tabular}

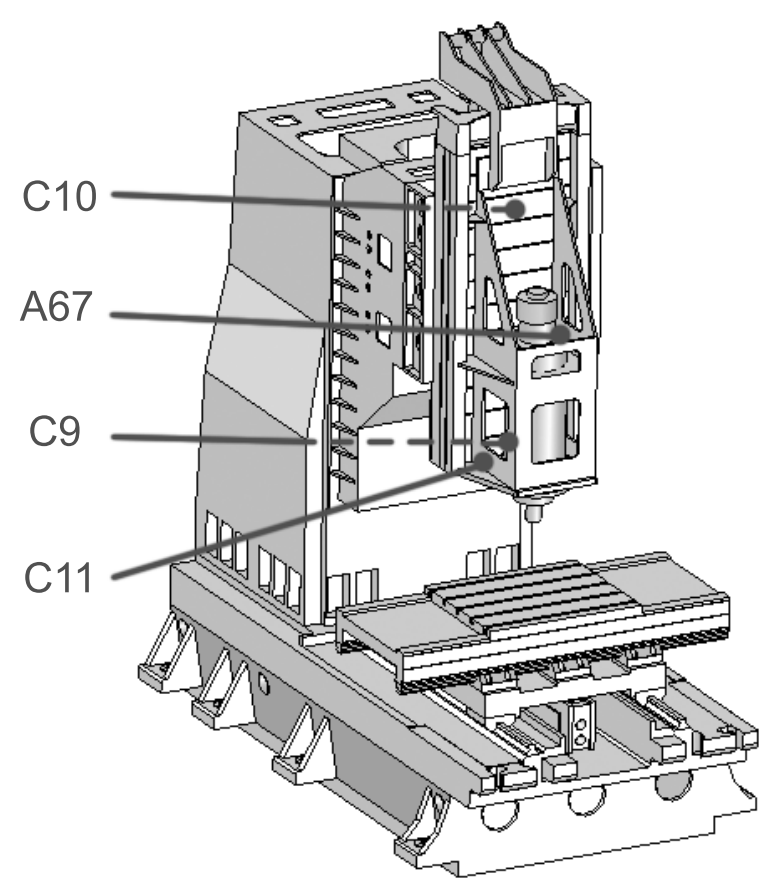

Fig. 5: Chosen sensors placement

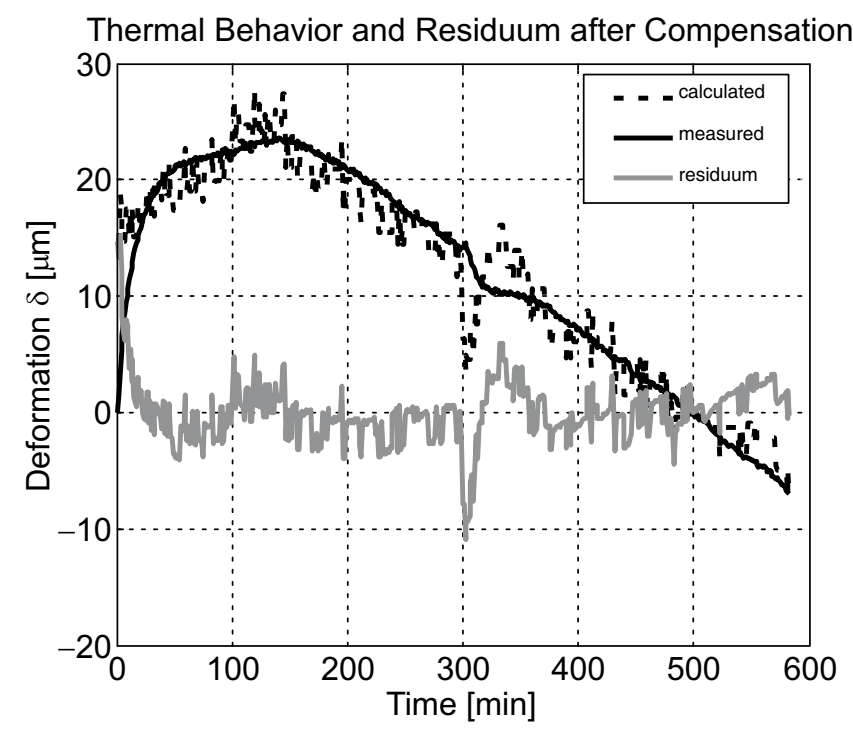

Fig. 6: First thermal measurement 
The resulting deformation equation for the Z-axis for four inputs has the following form:

$$
\begin{aligned}
\delta_{Z} & =236.81-0.27 \times T_{1}-11.93 \times T_{2} \\
& -23.98 \times T_{3}+26.85 \times T_{4}
\end{aligned}
$$

This equation was determined by calculating the deformation and temperature tract during the first machine thermal behavior analysis, Fig. 6 . The calculation was checked by another machine measurement, with different initial conditions. The machine was in a different initial thermal state, due to the different room temperature. In addition, the frame itself was in a semi-warm state, due to incomplete cooling down from the previous working day. The cooling process had taken place only overnight, which is not long enough for this type of machine. The thermal load of the spindle was the same as in the first analysis.

\section{Compensation results}

The residual deformation after compensation is shown in Fig. 7. Clearly, the applied compensation has a positive influence. A good improvement can be seen in the middle transient phase, where the influences of the spindle deformation and of the column deformation are opposite. It is always difficult to describe this phase, because the superposition of the two deformations plays a significant role. Also, during the first phase, when the big spindle deformation takes place, we can see the good quality of the compensation mechanism. There is a very fast increase in the spindle deformation. Multinomial compensation with spindle cooling liquid measurement eliminates this effect in a shorter time than without compensation.

The principle of multinomial regression calculation, together with the small number of sensors that have to be installed, limits the reaction speed when there are unexpected changes in machine behavior. This effect can be seen in Fig. 6 . around time "470 min". A sudden spindle cooling failure causes deformation variation. The compensation mechanism reacts, but not sufficiently. This is due to the sensors that are included in the compensation calculation. To improve this compensation type, a special multinomial approach is necessary for the spindle.

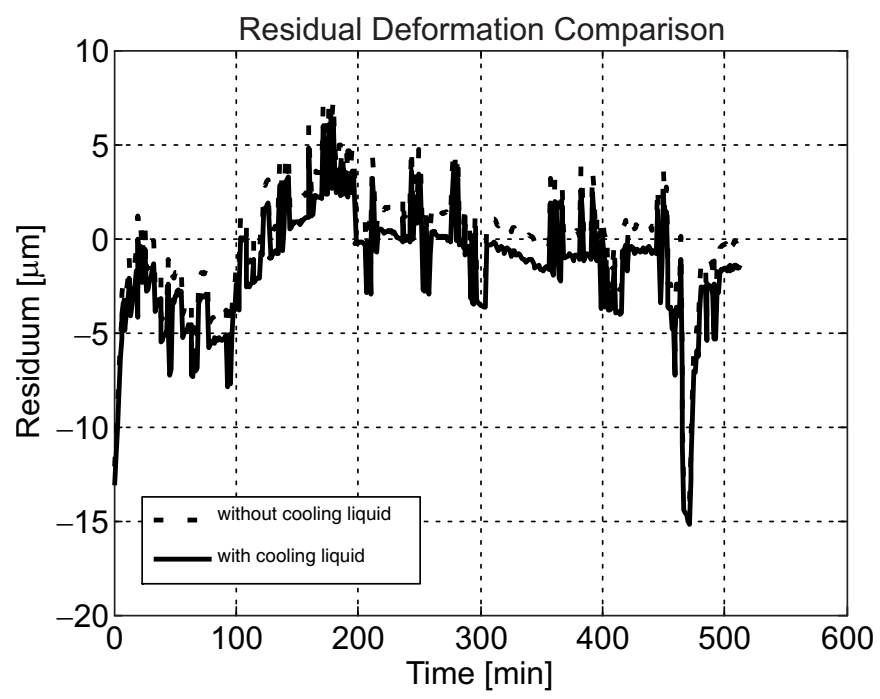

Fig. 7: Residual deformation after compensation

\section{Conclusion}

Using the spindle cooling liquid improves the multinomial regression compensation mechanism. The resulting residual deformation of the MCFV 5050LN machining center, in the $\mathrm{Z}$-axis, is better than when a standard regression calculation is made, using only a machine frame temperature measurement. The deformation can be eliminated faster, in the critical first phase. Because the calculation is compounded from four sensors: the machine frame, the spindle and cooling liquid, the reaction of the compensation is not fast enough for special unexpected events such as a cooling failure.

\section{Acknowledgment}

This research has been supported by the Ministry of Education of the Czech Republic - project 1M6840770003.

\section{Reference}

[1] Vyroubal, J.: Elimination of Thermal Deformation Due to CNC Machine Tool Spindles. In: $4^{\text {th }}$ International Conference and Exhibition on Design and Production of Machines and Dies/Molds, Middle East Technical University, 2007, p. 251-257.

[2] Ko, T. J. et al.: Particular Behavior of Spindle Thermal deformation by Thermal Bending. IJMT, Vol. 43 (2003), p. 17-23.

[3] Ramesh, R. et al.: Thermal Error Measurement and Modeling in Machine Tools. Part I. Influence of Varying Operating Conditions. IJMT, Vol. 43 (2003), p. 391-404.

[4] Lo, Ch. H.: Optimal Temperature Variable Selection by Grouping approach for Thermal Error Modeling and Compensation. IJMT, Vol. 39 (1999), p. 1383-1396.

[5] Hornych, J., Vyroubal, J.: Inteligentni chladicí systém část I: tepelné chování izolované pinoly. Report V-07-055. Research Centre of Manufacturing Technology, 2007.

Ing. Jiří Vyroubal

phone: +420221990930

Fax: +420221990999

e-mail: j.vyroubal@rcmt.cvut.cz

Czech Technical University in Prague

Research Centre of Manufacturing Technology

Horská 3

128 00, Prague 2, Czech Republic 